\title{
The Navigation of Robotic Fiber Positioners in SDSS-V Project: Design and Implementation
}

\author{
Matin Macktoobian*, Denis Gillet ${ }^{\dagger}$, and Jean-Paul Kneib \\ Swiss Federal Institute of Technology in Lausanne (EPFL) \\ 1015 Lausanne, Switzerland \\ Emails: *matin.macktoobian@epfl.ch, †denis.gillet@epfl.ch, ${ }^{\ddagger}$ jean-paul.kneib@epfl.ch
}

\begin{abstract}
SDSS-V project is one of the major observational cosmological projects which aims to generate the map of the observable universe by collecting spectroscopic data from the sky using optical fibers. Each optical fiber is attached to a robotic positioner to be automatically coordinated. This paper illustrates the solution to the navigation problem of robotic fiber positioners corresponding to the SDSS-V project. We note the principal challenging requirements to navigate the robotic fiber positioners. These requirements are those which differentiate the navigation problem of the SDSS-V project from that of the other projects. Then, we discuss the solution in view of both design and implementation. In particular, we specify the hardware and the software components of the solution in a systematic perspective. We illustrate the effectiveness of our solution based on practical results.
\end{abstract}

Index Terms-robotic fiber positioners, SDSS-V, cosmological spectroscopy, navigation

\section{INTRODUCTION}

Electromagnetic waves emanated from cosmological objects have been taken into account as main sources of observational information exploited by ground and space telescopes. The Earth atmosphere is opaque with respect to many wavelengths of the cited spectrum, yet the visible light can penetrate through it and reach the Earth surface. This transparency makes optical fibers legit candidates to be used in many observations including the recent modern spectroscopic survey generations [1]. In particular, astronomy aims to the generation of a set of maps which represent the whole observable universe. For this purpose, a set of optical fibers are mounted at a specific plate of a ground telescope called focal plane. An observation includes a set of targets, say, galaxies, which have to be observed by the set of fibers. An algorithm, e.g., $[2,3]$, assigns a target to each fiber positioner. Then, fibers shall be coordinated such that each of them points to its assigned target. Subsequently, each fiber collects the visible light coming from its target during a particular exposure time. Finally, the received signals are sent to a spectrograph by which a unified map is generated corresponding to the whole objects of the observation. Since many observations are often planned to be successively taken into account, fibers have to be coordinated to point at their new targets corresponding to another observation. Thus, a robotic fiber positioner is attached

This work was financially supported by the Swiss National Science Foundation (SNF) grant number 20FL21_185771 and the SLOAN ARC/EPFL agreement number SSP523. to each fiber to automate the coordination process. The navigation of the whole robotic fiber positioners set associated with a particular observation is desired in spectroscopic observation projects.

Many instruments have been developed to realize cosmological spectroscopy and to obtain sky surveys. For example, the "Dark Energy Spectroscopic Instrument" (DESI) [4, 5] assesses the dark energy impact on the expansion of the universe using baryon acoustic oscillations technique. The DESI fiber system includes 5000 robotic positioners arranged in a hexagonal formation with a $10.4 \mathrm{~mm}$ distance between actuators. As another example, the "Multi Object Optical and Near-infrared Spectrograph for the VLT" (MOONS) [6] probes the near-infrared spectra of millions of stars and galaxies to study their archeology and evolution. The MOONS fiber system encompasses 1000 robotic fiber positioners in a similar hexagonal distribution where the distance between actuators is approximately $3 \mathrm{~cm}$.

The "Sloan Digital Sky Surveys" (SDSS) [7] project is under development to measure critical early phase of the history of the cosmos. In particular, SDSS-V project aims to equip the Sloan instrument with a new generation of robotic fiber positioners $[8,9]$. This paper reports the accomplishments corresponding to the navigation of the robotic fiber positioners associated with the SDSS-V project. To be specific, we focus on the design implications, the instrumental implementation, and the interpretation of the obtained results.

The remainder of the paper is organized as follows. Section II reviews the mechanical characterization of the robotic fiber postioners developed for the SDSS-V project. Section III explain the challenges in the navigation of the SDSS-V robotic fiber positioners in view of collision avoidance (see, Section III-A) and complete coordination (see, Section III-B) problems. Then, Section IV illustrates how the cited challenges are handled in view of both hardware (see, Section IV-A) and software (see, Section IV-B) perspectives. Section V finally represents and interprets a batch of results obtained from the application of the described approach.

\section{Mechanical Characterization of Robotic POSITIONERS}

We briefly introduce fibers and robotic fiber positioners used in cosmic spectroscopy. A fiber is a string medium at the tip of which a sensor detects a specific type of electromagnetic waves 


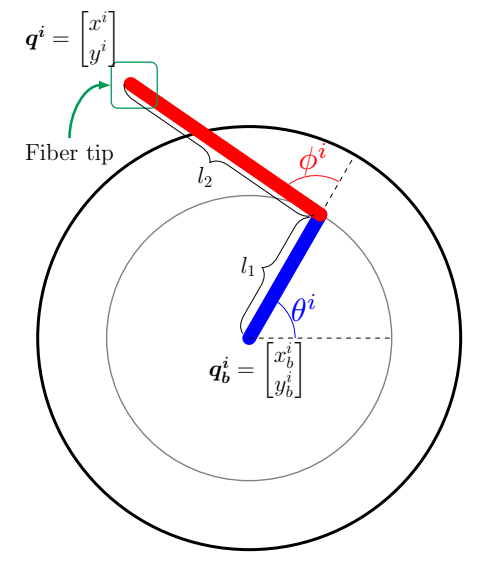

Fig. 1: The schematic of a typical robotic positioner

radiated by a particular object in the sky. Then, the signal is transmitted to a spectrograph, which processes it to add the trace of the observed object to the map it generates. Based on the typical application of fibers in cosmological observation, a swarm of fibers yields massive spectroscopic surveys.

A set of robotic positioners are mounted on a particular area of its hosting telescope called focal plane. Each robotic positioner is a planar RR manipulator whose end-effector, called ferrule, shall reach the point at which its fiber has to observe an object based on a particular observation or application. The forward kinematics corresponding to the workspace of each positioner is described as below.

$$
\boldsymbol{q}^{\boldsymbol{i}}=\boldsymbol{q}_{\boldsymbol{b}}^{\boldsymbol{i}}+\left[\begin{array}{cc}
\cos \left(\theta^{i}\right) & \cos \left(\theta^{i}+\phi^{i}\right) \\
\sin \left(\theta^{i}\right) & \sin \left(\theta^{i}+\phi^{i}\right)
\end{array}\right]+\boldsymbol{l}
$$

Here the $i^{\text {th }}$ positioner is located at $\boldsymbol{q}^{\boldsymbol{i}}=\left[\begin{array}{ll}x^{i} & y^{i}\end{array}\right]^{\top}$ with respect to a universal frame attached to the focal plane of the host telescope. $\boldsymbol{q}_{\boldsymbol{b}}^{\boldsymbol{i}}=\left[\begin{array}{ll}x_{b}^{i} & y_{b}^{i}\end{array}\right]^{\top}$ is also the base coordination of the positioner. The lengths of rotational links are represented by $\boldsymbol{l}=\left[\begin{array}{ll}l_{1} & l_{2}\end{array}\right]^{\top}$. The angular positions of the $i^{\text {th }}$ positioner are denoted by $\theta^{i}$ and $\phi^{i}$. The quoted parameters are depicted in fig. 1. Fig. 2 illustrates an arrangement of positioners placement in the focal plane of a typical telescope.

The axes of both rotational arms of a positioner are tilted with respect to the axis of its first rotational arm according to some particular specifications. The ferrule is also deviated with respect to the cited axis. So, when the positioner rotates its ferrule, the swept area is vaster than the area corresponding to the base of the positioner.

\section{SDSS-V NAVIGATION CHALLENGES}

\section{A. Collision Avoidance for Dense Robotic Positioners Sets}

To increase the throughputs of observations, SDSS-V project requires a dense formation of robotic fiber positioners per area of focal planes compared to the former projects. This requirement is addressed by developing a new generation of robotic fiber postioners whose overall diameters are $25 \mathrm{~mm}$ and $7 \mathrm{~mm}$. Thus, the distance between adjacent robotic fiber

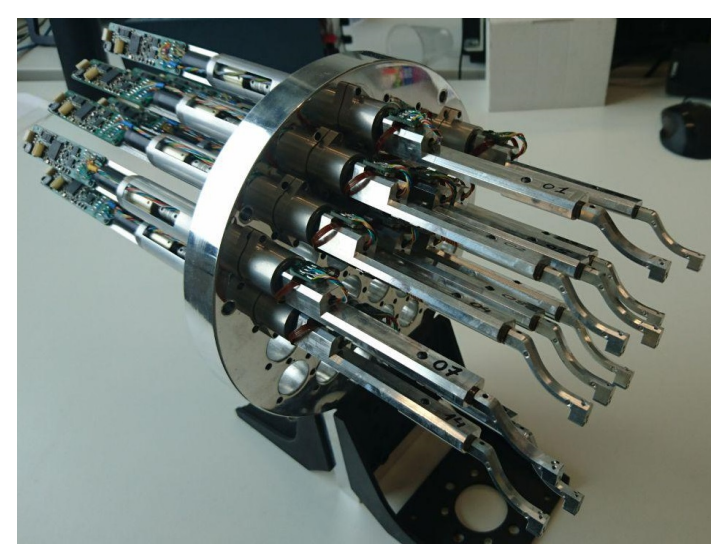

Fig. 2: A set of robotic fiber positioners mounted on a typical focal plane

positioners can be extremely decreased. However, the collision avoidance problem becomes more challenging compared to that of the systems with less density of robotic fiber positioners. So, the desired navigation approach has to consider a variety of mechanical constraints such as fiber torsion and tension to avoid potential collisions.

\section{B. Completeness Seeking}

The working spaces of adjacent robotic fiber positionrs are highly overlapping with each other because of their proximity and hexagonal arrangement. So, the complete coordination of robotic fiber positioners becomes a complicated issue. In particular, a coordination associated with a specific observation is complete if all of the robotic fiber positioners involved in the observation reach their target spots at the end of the navigation process. The former projects had achieved partial completeness [10] with high rates of convergence. Yet, complete coordination is an important performance requirement to be fulfilled.

\section{NAVIGATION APPROACH}

Our navigation strategy constitutes a hardware subsystem which represents the overall robotic fiber positioners. A software subsystem is also taken into account to compute the trajectories of robotic fiber positioners while collisions are avoided.

\section{A. Hardware Architecture}

Each robotic fiber positioner is equipped with an on-board processing unit which controls the motors of the robotic fiber positioner based on trajectories received from a computer. The overall hardware architecture of an on-board system is illustrated in fig. 3. In particular, an STM32 microcontroller supervises the functionality of each robotic fiber positioner. Two motors are mounted on each robotic fiber positioner to move its arms. A computer calculates the trajectories according to which the arms of the robotic fiber positioner need to rotate based on the specification of a particular observation. 


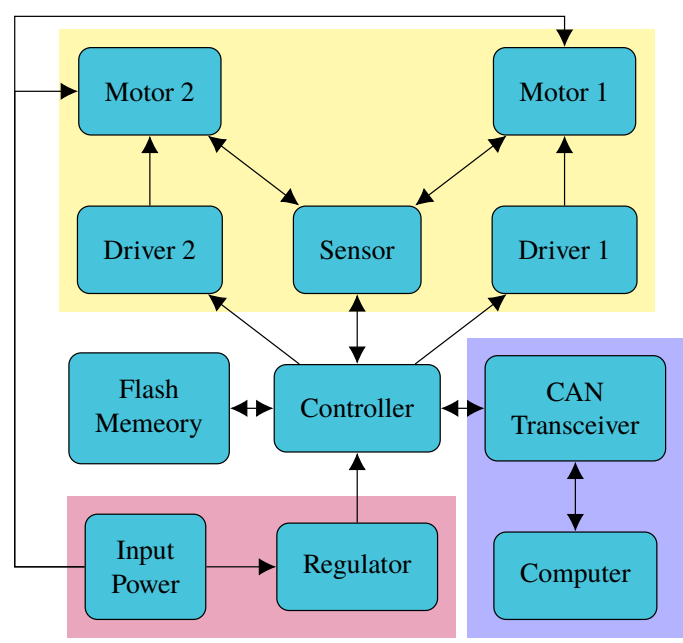

Fig. 3: The hardware architecture of the on-board system corresponding to a typical robotic fiber positioner

Then, the trajectories are transferred to the on-board system via CAN bus. For this purpose, a CAN transceiver is taken into account to realize the CAN protocol in the system. The internal memory of the microcontroller is limited. Thus, an external flash memory is added to the architecture for specific computational purposes. For example, the linearization of the dynamics of the coupled mechanical parts requires a set of intermediate points used during the runtime of the system which can be saved in the flash memory. Furthermore, the flash memory can host some local configurations of the system in the future. Finally, a sensor is also taken into account to capture the angular velocity corresponding to the tip of the robotic fiber positioner's ferrule.

The ferrule of the recently developed robotic fiber positioner supports up to 3 fibers. Thus, the next generation of multifiber spectroscopic surveys can efficiently benefit from these instruments.

\section{B. Software Architecture}

The generation of arms trajectories is done by a coordination software unit which has to be run on an external computing unit. The two-level architecture of the quoted software is depicted in fig. 4. In particular, the low-level navigator is a cooperative artificial potential function which seeks the collision-free complete coordination of all robotic fiber positioners corresponding to a hexagonal neighborhood. A high-level finite state machine is also considered at the top of the cited low-level navigator. This finite state machine makes decisions regarding complicated coordination scenarios including deadlocks and similar priorities, which cannot be properly handled by the low-level navigator. The coordination software returns a YAML file including two arrays of positional entries corresponding to each robotic fiber positioner. Given a particular robotic fiber positioner, each of its YAML

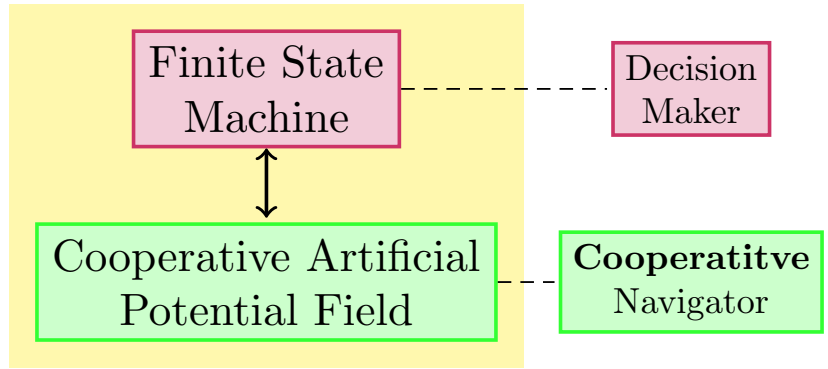

Fig. 4: The architecture of the coordination software

file's arrays are associated with one of its arms. Since the time interval between successive entries is already known according to the observation specification, the entries represent the trajectories associated with their robotic fiber positioners over the passage of time. The algorithm takes into account the mechanical constraints including torsion on fibers, the avoidance zones based on soft and hard stops, and mechanical specifications of robotic fiber positioners like torsion. The overall behavior of the system is verified using meteorology cameras.

The traditional approach to using artificial potential fields for the purpose of navigation often applies an artificial potential field to each robotic fiber positioner in which only its convergence is considered. This competitive perspective practically gives rise to partially-complete coordinations since each positioner only cares about its own convergence. Instead, we propose a cooperative artificial potential field whose attractive part takes into account not only the convergence of its particular robotic fiber positioner but also those of its adjacent peers. Thus, one obtains the necessary condition to achieve the complete coordination using cooperative artificial potential fields.

\section{RESUlts}

A typical simulation scenario including 15 robotic fiber positioners is taken into account to illustrate the functionality of the proposed navigation process as depicted in fig. 5 . Major parameters of the simulation are specified in the table I. In particular, fig. 5a illustrates the initial positions of the hypothetical robotic fiber positioners. The red circles represent the target spots associated with a hypothetical observation. The navigation results is depicted in fig. $5 \mathrm{~b}$. The evolution of the convergence is collision free, and one notes that all of the robotic fiber positioners reach their target positions, thereby obtaining complete coordination.

TABLE I: The parameters applied to the simulation

\begin{tabular}{ll} 
Parameter & Value \\
\hline The length of the first arm & $8.000 \mathrm{~mm}$ \\
The length of the second arm & $17.000 \mathrm{~mm}$ \\
The width of the first arm & $8.000 \mathrm{~mm}$ \\
The width of the second arm & $4.000 \mathrm{~mm}$ \\
\hline
\end{tabular}




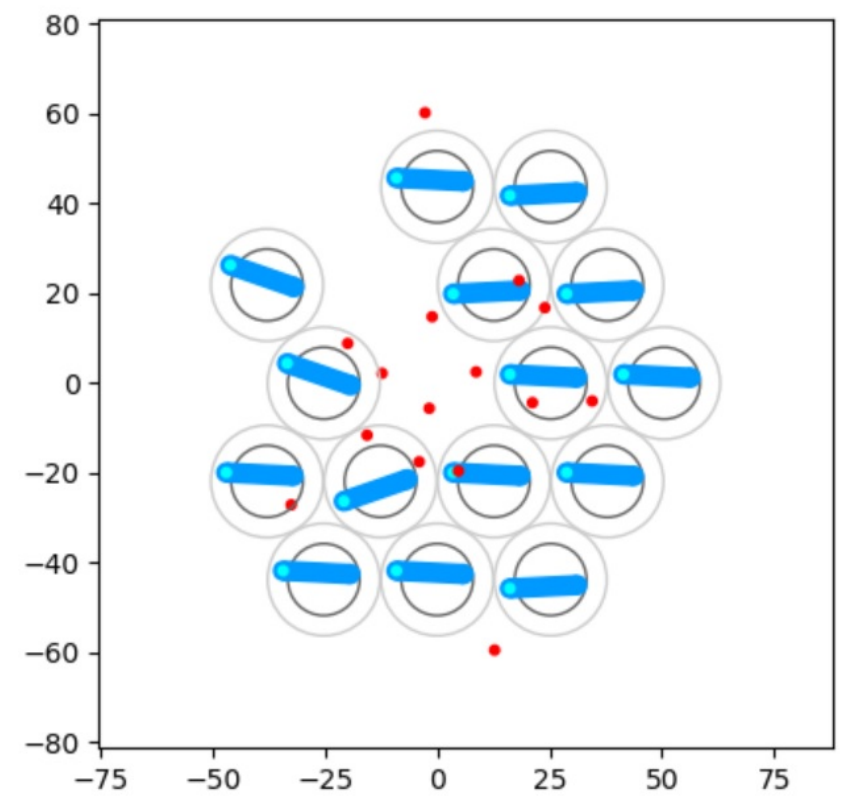

(a) The initial condition of an uncoordinated set of robotic fiber positioners

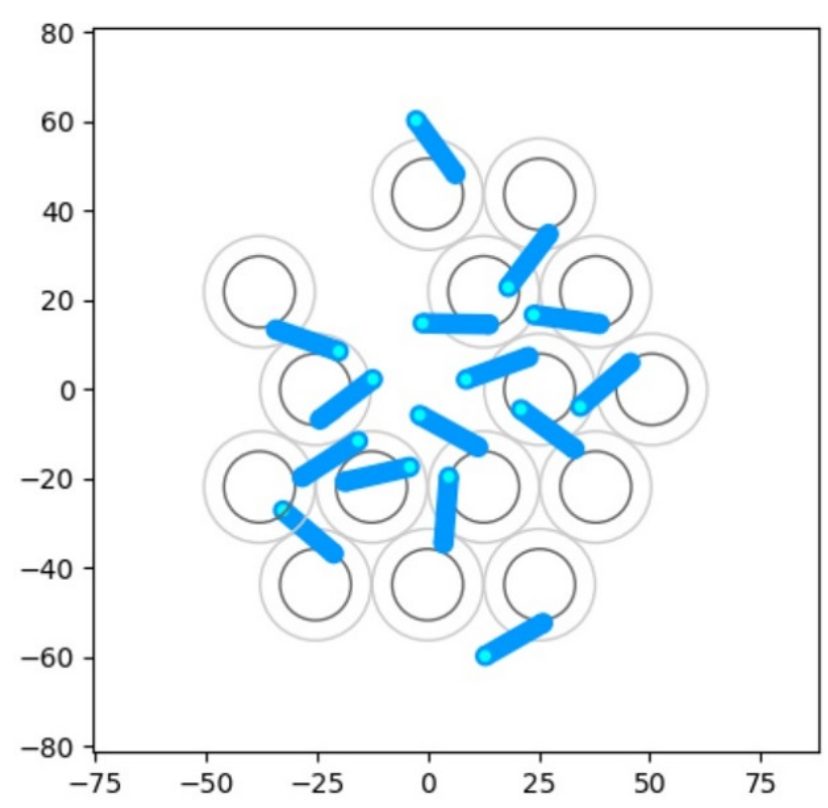

(b) The completely coordinated result of the navigation process

Fig. 5: A complete coordination scenario using the proposed navigation solution

\section{REFERENCES}

[1] E. Mazets, S. Golenetskii, V. Ilyinskii, Y. A. Guryan, R. Aptekar, V. Panov, I. Sokolov, Z. Y. Sokolova, and T. Kharitonova, "Cosmic gamma-ray burst spectroscopy," Astrophysics and Space Science, vol. 82, no. 2, pp. 261282, 1982.

[2] I. Morales, A. D. Montero-Dorta, M. Azzaro, F. Prada, J. Sánchez, and S. Becerril, "Fibre assignment in nextgeneration wide-field spectrographs," Monthly Notices of the Royal Astronomical Society, vol. 419, no. 2, pp. 1187-1196, 2011.

[3] C. E. Schaefer, L. Makarem, and J.-P. Kneib, "Targetbased fiber assignment for large survey spectrographs," in Software and Cyberinfrastructure for Astronomy IV, vol. 9913, p. 991335, International Society for Optics and Photonics, 2016.

[4] A. Aghamousa, J. Aguilar, S. Ahlen, S. Alam, L. E. Allen, C. A. Prieto, J. Annis, S. Bailey, C. Balland, O. Ballester, et al., "The desi experiment part i: Science, targeting, and survey design," arXiv preprint arXiv:1611.00036, 2016.

[5] A. Aghamousa, J. Aguilar, S. Ahlen, S. Alam, L. E. Allen, C. A. Prieto, J. Annis, S. Bailey, C. Balland, O. Ballester, et al., "The desi experiment part ii: Instrument design," arXiv preprint arXiv:1611.00037, 2016.

[6] M. Cirasuolo, J. Afonso, M. Carollo, H. Flores, R. Maiolino, E. Oliva, S. Paltani, L. Vanzi, C. Evans, M. Abreu, et al., "Moons: the multi-object optical and near-infrared spectrograph for the vlt," in Ground-based and airborne instrumentation for astronomy V, vol. 9147, p. $91470 \mathrm{~N}$, International Society for Optics and Photonics, 2014.

[7] D. G. York, J. Adelman, J. E. Anderson Jr, S. F. Anderson, J. Annis, N. A. Bahcall, J. Bakken, R. Barkhouser, S. Bastian, E. Berman, et al., "The sloan digital sky survey: Technical summary," The Astronomical Journal, vol. 120, no. 3, p. 1579, 2000.

[8] P. Hörler, L. Kronig, J.-P. Kneib, M. Bouri, H. Bleuler, and D. von Moos, "High density fiber postitioner system for massive spectroscopic surveys," Monthly Notices of the Royal Astronomical Society, vol. 481, no. 3, pp. 3070-3082, 2018.

[9] P. Hörler, "Robotic fiber positioning systems for massive spectroscopic surveys: Mechanical design guidelines and technological opportunities," tech. rep., EPFL, 2018.

[10] D. Tao, L. Makarem, M. Bouri, J.-P. Kneib, and D. Gillet, "Priority coordination of fiber positioners in multi-objects spectrographs," in Ground-based and Airborne Instrumentation for Astronomy VII, vol. 10702, p. $107028 \mathrm{~K}$, International Society for Optics and Photonics, 2018.

[11] "Moons: Multi-object optical and near-ir spectrograph." https://vltmoons.org/technology/spectrograph/. Accessed: 2019-02-12.

[12] S. Doeleman, E. Agol, D. Backer, et al., "Astro2010: The astronomy and astrophysics decadal survey," Science White Papers, vol. 68, 2009. 